\title{
Conhecimento dos farmacêuticos atuantes em drogarias e farmácias sobre a prescrição farmacêutica na cidade de Mineiros-Goiás
}

\author{
Rafaella Ferreira Coelho' e Fabio Bahls Machado²
}

\author{
1 Farmacêutica, graduada na Faculdade Morgana Potrich - FAMP, Brasil. \\ E-mail: rafaellafc@outlook.com http://lattes.cnpq.br/9342496552263301 id http://orcid.org/0000-0002-2078-6050 \\ 2 Doutorando em Ciências Farmacêuticas pela UFG, Mestre em Ciências Farmacêuticas pela UEM, Especialista em Farmacologia pela \\ UNICESUMAR e Especializando em Docência do Ensino Superior pela FAMP. Professor na Faculdade Morgana Potrich, Brasil. \\ Email: famafarm@yahoo.com.br $\underline{\text { http://lattes.cnpq.br/3864362390361071 id http://orcid.org/0000-0001-7688-8040 }}$
}

RESUMO: O farmacêutico é o profissional que tem competência para realizar a dispensação de medicamentos, a partir da prescrição e de outros serviços prestados. Neste sentido o objetivo do trabalho foi verificar o conhecimento dos farmacêuticos referente a prescrição farmacêutica. Foram visitadas um total de 31 drogarias e 04 farmácias de manipulação da cidade de Mineiros-Goiás no período de 25 de julho a 15 de agosto de 2017, sendo que destas somente 17 profissionais participaram. A partir da coleta de dados nota-se que em $42,86 \%$ dos estabelecimentos visitados os profissionais se encontraram ausentes. A principal causa de não se especializar em prescrição farmacêutica e/ou farmácia clínica com 35,29\%, é a falta de tempo. Notase que $76,47 \%$ dos profissionais se consideram habilitados a realizar a prescrição, sendo que somente $23,52 \%$ desses possuem especialização em prescrição farmacêutica e/ou farmácia clínica. Conclui-se que é notável a ausência de um número significativo de profissionais nos estabelecimentos farmacêuticos, bem como a incoerência referente a estes presumirem estarem habilitados a exercerem a prescrição, dado que apesar de um número considerável de profissionais demonstrarem conhecimento com relação RDC do CFF 586/2013, somente uma minoria tem uma base preparatória propícia por meio de especialização em prescrição farmacêutica e/ou farmácia clínica.

Palavras-chave: Atenção Farmacêutica. Semiologia. Medicamentos.

\section{Knowledge of pharmacists acting in drug stores and pharmacies} about pharmaceutical prescription in the city of Mineiros-Goiás

ABSTRACT: The pharmacist is the professional who has the competence to carry out the dispensation of medicines, from prescription and other services provided. On this way, the goal of this work was to verify the pharmacists' knowledge regarding pharmaceutical prescription. A total of 31 drug stores and 4 manipulation pharmacies were visited in the city of Mineiros-Goiás from July 25 to August 15 in 2017, of which only 17 professionals participated. From the data it can be noted that in $42.86 \%$ of the visited establishments the professionals were absent. The main reason of not specializing in pharmaceutical prescription and / or clinical pharmacy in $35.29 \%$ of cases, is the lack of time. It was observed that $76.47 \%$ of professionals consider themselves qualified to carry out the prescription, however only $23.52 \%$ of these have specialization in pharmaceutical prescription and / or clinical pharmacy. It was concluded that the absence of a significant number of professionals in pharmacies is notable, as well as the incoherence of those who presume to be able to do the prescription. despite the fact that a considerable number of professionals demonstrate knowledge regarding about RDC do CFF 586/2013, only a minority has a favorable preparatory base through specialization in pharmaceutical prescription and / or clinical pharmacy.

Keywords: Pharmaceutical attention. Semiology. Medicines. 


\section{INTRODUÇÃO}

A Farmácia Clínica originou-se a partir do surgimento das indústrias farmacêuticas. Sendo assim, houve a necessidade da implantação da Atenção Farmacêutica (ATENFAR), dado a banalização da utilização dos medicamentos e o consequente desprestígio dos profissionais farmacêuticos (BISSON, 2007). A ATENFAR é proveniente da necessidade de uma interação do farmacêutico para com o paciente utente da terapia farmacológica, a fim de orientar quanto ao uso racional de medicamentos e prevenir os problemas causados por estes (PEREIRA; FREITAS, 2008).

É importante salientar que o medicamento tem finalidade profilática e curativa sendo este uma das principais medidas para o reestabelecimento e preservação da saúde. Entretanto, seu uso indevido além de ser dispendioso é um dos principais problemas de saúde pública (VIEIRA, 2007). Em média $50 \%$ dos fármacos são fornecidos e consumidos de forma inapropriada. No Brasil, cerca de $35 \%$ dos medicamentos comprados nos estabelecimentos de saúde são de escolha própria do paciente, sem a orientação de um profissional adequado, o que pode gerar graves problemas de saúde. O uso de uma farmacoterapia inadequada, corresponde à $27 \%$ das intoxicações por medicamentos e em consequência 16\% destas podem evoluir para óbito (AQUINO, 2008).

O farmacêutico é o profissional que está diretamente vinculado ao medicamento, objetivando minimizar ou reverter as consequências acarretadas a saúde em virtude do mau uso destes (VIEIRA, 2007). Já o farmacêutico clínico, tem suas atribuições regidas pela Resolução do Conselho Federal de Farmácia (CFF) 585/2013 e, a prescrição farmacêutica está amparada na Resolução $586 / 2013$ do CFF. Onde, esta é uma atribuição clínica do farmacêutico, e, que está em evidência. Sendo realizada de acordo com a história e condição clínica do paciente (CFF, 2013a).

A RDC no 586/2013 regulamenta a prescrição farmacêutica e em consequência busca o melhor tratamento farmacoterapêutico ou terapias alternativas, que visem a qualidade de vida do paciente, de forma individualizada. O profissional poderá prescrever os medicamentos que não exijam receita médica baseado nas condições clínicas do paciente, minimizando assim a ocorrência de efeitos adversos e o uso irracional de medicamentos. Os farmacêuticos habilitados a prescreverem, de acordo com a Resolução $586 / 13$, além da formação convencional devem estar respaldados em uma especialização voltada para a área clínica que dê o embasamento necessário para identificação dos problemas relacionados a saúde, visando assim um tratamento farmacoterapêutico seguro e eficaz (CRF-MG, 2013).

O farmacêutico clínico tem competência para realizar a prescrição farmacêutica, sendo esta, uma forma de enriquecer a busca por um tratamento que objetive 0 bem-estar do indivíduo, mediante a escolha de uma farmacoterapia eficaz, individual e acessível ao paciente, ou, até mesmo por meio do reconhecimento da necessidade do auxílio de outros profissionais da saúde (CFF, 2013c).

Neste sentido, a presente pesquisa tem como fundamento analisar a percepção do conhecimento dos farmacêuticos face a prescrição farmacêutica, tendo como propósito informar à população e o público 
acadêmico sobre a importância da prescrição farmacêutica voltada para a saúde da população que faz uso de tratamento farmacológico.

\section{METODOLOGIA}

\subsection{Caracterização do estudo}

Trata-se de uma pesquisa exploratória quantitativa, onde o levantamento de dados efetuou-se por meio de entrevistas realizadas com os farmacêuticos presentes nas drogarias e farmácias da cidade de Mineiros, estado de Goiás.

\subsection{Procedimento de coleta de dados}

A cidade de Mineiros-GO conta com quatro farmácias de manipulação e trinta e quatro drogarias, segundo os dados fornecidos pelo CRF-GO. Porém, foram encontrados um total de 31 drogarias e quatro farmácias de manipulação.

É importante salientar que foram respeitados todos os princípios éticos e morais da pesquisa, conforme a Resolução 466/2012 do Conselho Nacional de Saúde (CNS) e Comissão Nacional de Ética em Pesquisa (CONEP).

O projeto foi submetido ao Comitê de Ética e Pesquisa em Seres Humanos das Faculdades Integradas de Santa Fé do Sul FISA/FUNEC em 23/05/2017 e aprovado no dia 25/07/2017, sob o número 687715 17.8.0000.5428. Posteriormente à aprovação do Comitê de Ética, iniciou-se a pesquisa mediante a aplicação dos questionários aos profissionais farmacêuticos da cidade de Mineiros-Goiás no período de 25/07/2017 à 15/08/2017. Os questionários foram compostos por nove questões estru- turadas e uma semiestruturada.

O público alvo da pesquisa foram os profissionais farmacêuticos atuantes nas farmácias e drogarias da cidade de MineirosGoiás. Estes profissionais foram abordados individualmente, onde foi esclarecido os objetivos e finalidade da pesquisa e então estes foram convidados a participarem da pesquisa, tendo o poder de escolha quanto a sua participação. Os profissionais que aceitaram colaborar com a pesquisa realizaram a leitura da Carta de Informação ao Participante da Pesquisa, posteriormente assinaram esta e o Termo de Consentimento Livre e Esclarecido, assegurando sua participação e sigilo quanto às informações prestadas. Durante a aplicação do questionário somente um profissional desistiu de participar da pesquisa expondo não ter conhecimento quanto as questões específicas. Foram aplicados um total de dezessete questionários, dado a recusa de dois profissionais, desistência de um e ausência de quinze outros. Considerou-se ausente após, os pesquisadores visitarem as farmácias pelo menos duas vezes no local e não encontrar o farmacêutico responsável técnico pelo estabelecimento.

\subsection{Análises de dados}

Os dados levantados foram analisados após a visita e aplicação dos questionários, os quais foram submetidos a análise mediante gráficos, por meio do Microsoft Excel, visando a organização dos dados para meIhor compreensão dos resultados.

\section{RESULTADOS E DISCUSSÃO}

O gráfico 1 demonstra que foram visitados um total de 35 estabelecimentos, nos 
quais 31 foram drogarias e quatro farmácias de manipulação. Sendo que, dezessete $(48,57 \%)$ farmacêuticos responderam o questionário, porém, um número significante de farmacêuticos, composto por quinze $(42,86 \%)$ profissionais encontraram-se ausentes nos estabelecimentos, do qual quatorze estavam ausentes nas drogarias $\mathrm{e}$ apenas um farmacêutico se encontrou ausente na farmácia de manipulação no momento da visita. Durante a aplicação do questionário apenas um (2,86\%) profissional desistiu de responder a pesquisa, manifestando não ter conhecimento sobre o assunto e dois $(5,71 \%)$ profissionais se recusaram a participar da pesquisa.

Gráfico 1. Porcentagem de farmacêuticos presentes nas drogarias e farmácias de manipulação da cidade de Mineiros-Goiás. Figure 1. Percentage of pharmacists in drugstores and manipulation pharmacies in the city of Mineiros-Goiás.

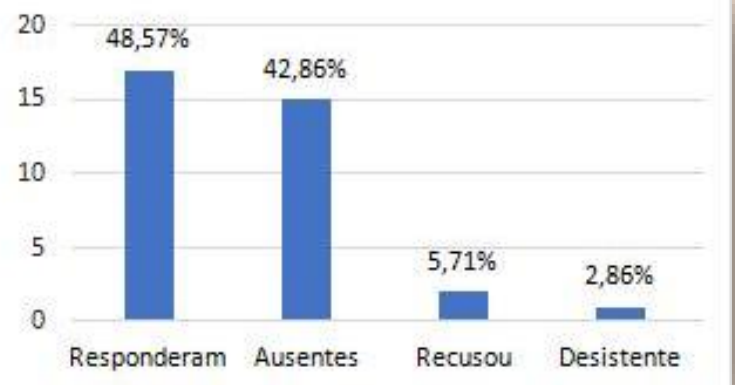

Fonte: Próprios pesquisadores.

O uso exacerbado de medicamentos é responsável por inúmeras complicações à saúde. Sendo assim, é notável a imprescindibilidade do farmacêutico atuante nos diversos estabelecimentos de saúde, visto que, este é o profissional que colabora para o restabelecimento do bem-estar do usuário de medicamentos, por meio da atenção farmacêutica (VIEIRA, 2007).

O farmacêutico não é um profissional que realiza somente a dispensação, seu trabalho envolve uma sequência de ações nas quais o principal beneficiário é o paciente, isso ocorre, por meio da análise de diversas informações, que são relevantes na seleção do tratamento terapêutico. Com a inserção da farmácia clínica a população desfruta de um profissional preparado para ampará-lo, contribuindo para a escolha do tratamento adequado de acordo com sua necessidade, visando a saúde, buscando minimizar o uso indiscriminado dos fármacos elegendo aquele que seja preciso à condição de saúde de forma individualizada (SANTOS, 2009).

A presença dos farmacêuticos legalmente habilitados nas farmácias e drogarias é um ato compulsório, visto que, através da prestação da ATENFAR, por meio de um diálogo com o paciente, o farmacêutico não só poderá identificar a condição de saúde como encaminhar o paciente quando julgar necessário a um serviço médico, evitando assim a administração desnecessária de determinados medicamentos (JOTZ et al., 2002).

Segundo a lei no 13.021 , de 8 de agosto de 2014, a presença do farmacêutico nos estabelecimentos de saúde é indispensável, visto que compete a este profissional garantir ao usuário de medicamentos um serviço de qualidade, por meio da seleção do tratamento terapêutico efetivo bem como instruí-lo (BRASÍLIA, 2014).

As duas primeiras colunas do gráfico 2 expressam que um total de sete profissionais que atuam em um período de até um ano como farmacêutico em drogarias e farmácias de manipulação, sendo que destes apenas um possui especialização, porém, não é em farmácia clínica e/ou prescrição farmacêutica. A terceira coluna, ex- 
pressa um total de três profissionais atuantes no período entre cinco a dez anos como farmacêutico em farmácias e drogarias, sendo que todos possuem especialização, porém, somente um possui especialização em farmácia clínica e/ou prescrição farmacêutica. Já a quarta e quinta colunas expressam que um total de sete farmacêuticos atuantes em um período superior a dez anos como farmacêutico em farmácias e drogarias, sendo que cinco possuem especialização e destes somente três é em farmácia clínica e/ou prescrição farmacêutica e dois não possuem nenhum tipo de especialização.

Gráfico 2. Tempo de Experiência do farmacêutico responsável e relação com atualização profissional.

Figure 2. Pharmacist's time of experience and Relationship with Professional Update.

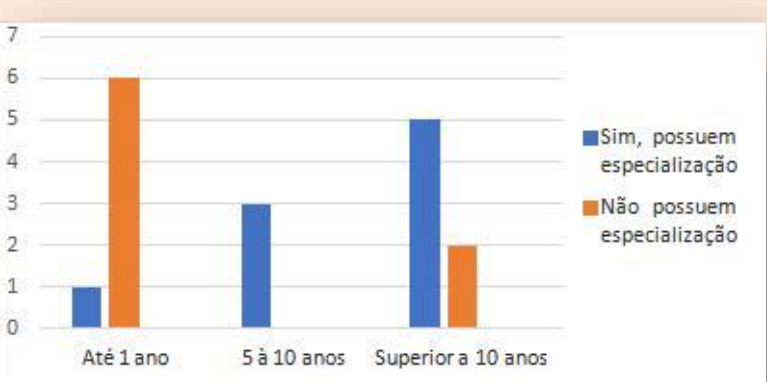

Fonte: Próprios pesquisadores.

Constata-se que o índice de farmacêuticos que não possuem especialização corresponde à oito $(47,06 \%)$ dos profissionais entrevistados, porém, não foi investigada a causa desta restrição nesses profissionais com relação ao aprimoramento dos seus conhecimentos, entretanto acredita-se que uma das causas talvez, seja, a falta de tempo, já que, quando questionados sobre a principal dificuldade em especializar em prescrição farmacêutica, seis $(35,3 \%)$ dos profissionais disseram ser por falta de tem- po.

Já os profissionais que possuem especialização correspondem a nove (52,94\%), sendo que as áreas de domínio são: farmácia clínica e/ou prescrição farmacêutica (23,53\%); saúde pública $(11,77 \%)$; farmácia magistral $(5,88 \%)$ e bioquímica $(5,88 \%)$.

É importante ressaltar que a qualificação dos farmacêuticos amplia a credibilidade destes profissionais que, através da ampliação dos seus conhecimentos se mostram autoconfiantes para o exercício da profissão, tendo como meta o bem-estar e saúde do usuário de medicamentos (PADUAN et al., 2005).

O gráfico 3 expressa que sete (41,18\%) profissionais possuem dificuldade em se especializar em prescrição devido: à ausência de um local apropriado que reserve as informações confidenciais coletadas no ato do atendimento; seis $(35,29 \%)$ profissionais não dispõem de tempo suficiente para se qualificar; três $(17,65 \%)$ não se especializam por outros motivos; um $(5,88 \%)$ respondeu nenhuma das alternativas e nenhum dos profissionais disseram ser por falta de conhecimento desta nova atribuição.

Gráfico 3. Exibe a dificuldade para a adequação em prescrição farmacêutica.

Figure 3. Shows the difficulty for suitability in pharmaceutical prescription.

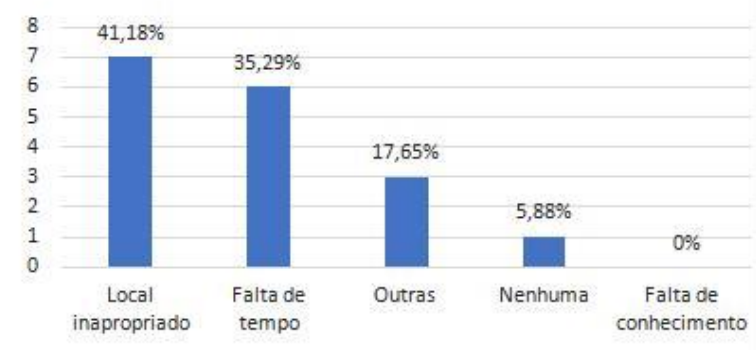

Fonte: Próprios pesquisadores. 
Silva e Lucca (2015) em sua pesquisa sobre a prescrição farmacêutica uma análise da percepção dos profissionais farmacêuticos da cidade de Cascavel-PR, constataram que: dezessete (50\%) dos farmacêuticos manifestaram ausência de um espaço próprio que assegure o sigilo do relato do paciente; dezesseis $(47,06 \%)$ afirmaram dispor de local apropriado para a realização da prescrição e um $(2,94 \%)$ decidiu não se manifestar, ou seja, comparando os estudos realizados verifica-se que a maior motivação do não exercício da prescrição por parte dos farmacêuticos se dá por falta de local apropriado que garanta a privacidade dos pacientes o mesmo que foi encontrado na presente pesquisa.

Segundo a RDC da Anvisa 44/2009 para que sejam preservadas as informações do paciente, não é necessário que o estabelecimento de saúde disponha de um local específico para o atendimento, porém, devese garantir a proteção da confidencialidade do paciente (BRASIL, 2009).

O gráfico 4 evidencia que: treze $(76,47 \%)$ farmacêuticos se consideram habilitados a realizarem a prescrição farmacêutica; três $(17,65 \%)$ não se consideram capazes de realizar esta atividade e um (5,88\%) farmacêutico se mostrou inseguro para realizar a prescrição.
Gráfico 4. Expressa a porcentagem de farmacêuticos que se consideram habilitados a realizarem a prescrição farmacêutica. Figure 4. Percentage of pharmacists who consider themselves qualified to carry out the pharmaceutical prescription.

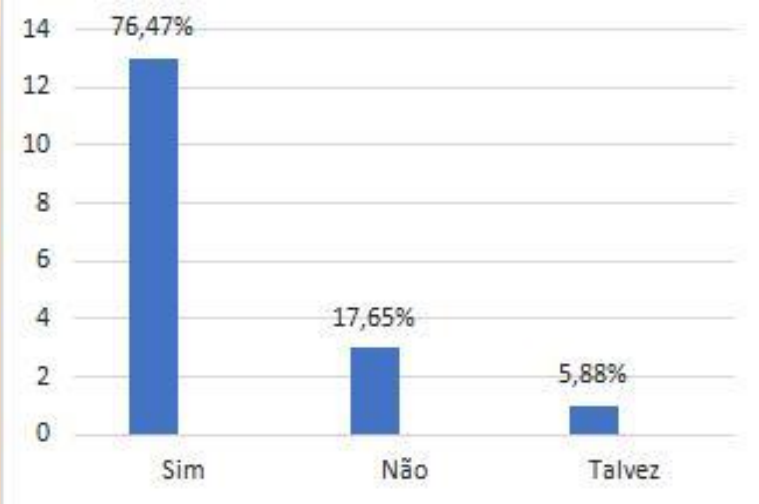

Fonte: Próprios pesquisadores.

Silva e Lucca (2015) em sua pesquisa semelhante, verificaram que: 27 (79,41\%) dos profissionais se consideram habilitados a realizarem a prescrição; cinco $(14,70 \%)$ não se consideram qualificados para realizarem a prescrição e dois $(5,89 \%)$ disseram que eventualmente se consideram habilitados, a depender da situação de saúde do paciente. Em comparação ao estudo de Silva e Lucca (2015) e a pesquisa atual, ambos os estudos demonstraram que a maioria dos entrevistados se consideram habilitados a realizarem a prescrição farmacêutica. Porém, é importante ressaltar que para exercer a prescrição farmacêutica, é fundamental não só se julgar habilitado a realizar este serviço como também estar em conformidade juntamente ao órgão responsável por fiscalizar o exercício da profissão e, ainda, ter domínio sob uma série de conteúdos os quais são essenciais para reconhecer e escolher o tratamento para as respectivas condições de saúde do usuário de medicamentos no que se refere a prescrição farmacêutica (CFF, 2013b). 
O gráfico 5 evidencia que: cinco $(29,40 \%)$ farmacêuticos realizam a ATENFAR somente quando solicitado; dez $(58,80 \%)$ farmacêuticos entrevistados realizam esta atividade constantemente e dois $(11,80 \%)$ farmacêuticos empregam esses serviços somente para grupos especiais.

Gráfico 5. Expressa a ocasião em que os profissionais realizam Atenção Farmacêutica.

Figure 5. Expresses the occasion in which the professionals realize Pharmaceutical Attention.

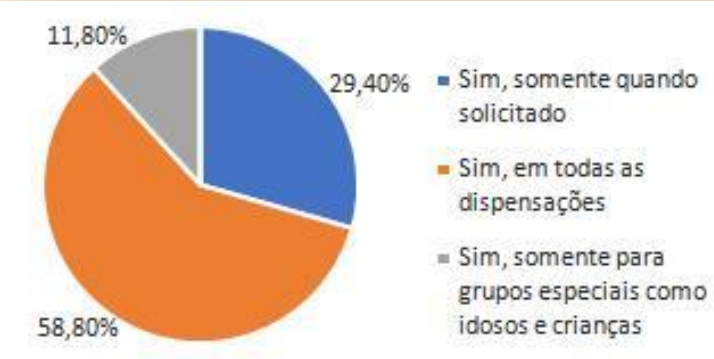

Fonte: Próprios pesquisadores.

A ATENFAR tem como principal propósito a promoção da saúde do usuário de medicamentos por meio de diversas ações, como por exemplo, a constatação de eventos indesejáveis que estejam associados aos fármacos usuais, tendo como finalidade atingir o restabelecimento da saúde. Porém, o farmacêutico enfrenta obstáculos quanto prestação de uma atenção eficiente ao usuário de medicamentos, em vista de que muitos proprietários de drogarias e farmácias objetivam o lucro, submetendo este profissional à realizar um atendimento rápido e muitas das vezes ineficaz, ou seja, este é desestimulado a realizar suas atividades que auxiliam na promoção da saúde da população, uma vez que, os lucros beneficiam os proprietários de estabelecimentos, e em consequência os pacientes ficam privados de um atendimento de melhor qualidade (OLIVEIRA et al., 2005).

A dispensação é uma atividade exclusiva do farmacêutico, porém, deve ser realizada de forma multidisciplinar juntamente com outros profissionais, visando precaver ou até mesmo medicar e tratar a condição de saúde do usuário de medicamentos. A dispensação não é somente a entrega dos medicamentos ao paciente, esta envolve a atenção à saúde, a anamnese e coleta de informações pertinentes ao estado de saúde, permitindo a seleção de um tratamento efetivo (GALATO, 2008).

O gráfico 6 exibe que dos farmacêuticos entrevistados quinze $(88,24 \%)$ realizam a indicação farmacêutica diariamente e dois $(11,76 \%)$ dos farmacêuticos indicam casualmente os medicamentos.

Gráfico 6. Frequência com que é realizada a indicação farmacêutica.

Figure 6. Frequency with which the pharmaceutical indication is made.

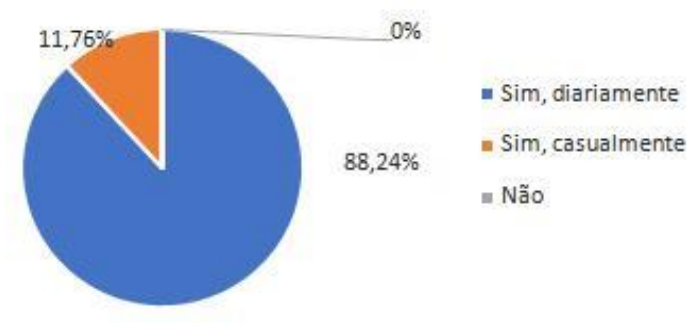

Fonte: Próprios pesquisadores.

A automedicação fundamenta-se principalmente do fácil acesso ao qual a população tem aos medicamentos, demonstrando assim a relevância do farmacêutico na liberação do medicamento proporcionando qualidade de vida ao usuário, conduzindo o tratamento do paciente da melhor forma possível, baseado na sua sintomatologia de 
forma individualizada (FERNANDES; CEMBRANELLI, 2015).

O farmacêutico por meio da prestação dos seus serviços evita a administração desnecessária de determinados medicamentos, principalmente daqueles que são de venda livre, os quais são os principais medicamentos responsáveis por causar complicações a saúde dos usuários (BORTOLON; KARNIKOWSKI; ASSIS, 2007).

O gráfico 7 evidencia que o serviço farmacêutico realizado em maior proporção nas farmácias e drogarias da cidade de Mineiros Goiás é a dispensação (quinze profissionais), em segundo lugar o acompanhamento farmacoterapêutico (nove profissionais) e a prescrição (nove profissionais), já a revisão da farmacoterapia é um dos serviços praticados em menor proporção (cinco profissionais) e alguns disseram realizar outros serviços (quatro profissionais). Vale ressaltar que, nestes dados o entrevistado pode responder mais de uma alternativa, 0 que inviabilizou a contabilização do percentual de cada alternativa respondida.

Gráfico 7. Serviços farmacêuticos realizados pelos entrevistados.

Figure 7. Pharmaceutical services performed by the interviewees.

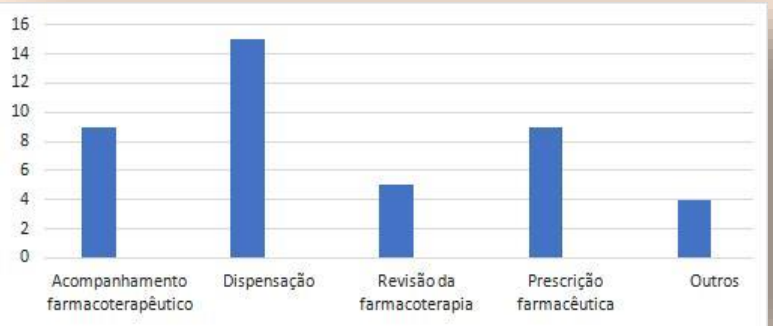

Fonte: Próprios pesquisadores.

Os serviços farmacêuticos são substancialmente importantes para a promoção da saúde da população, uma vez que, este, é o profissional capacitado para orientar o usuário de medicamentos, visando a redução de gastos públicos desnecessários (JOÃO, 2010). Dentre os serviços farmacêuticos realizados um dos mais importantes é a dispensação, em que o farmacêutico não só fornece o fármaco ao paciente, como também analisa a prescrição no que diz respeito aos aspectos legais, correlaciona a sintomatologia do paciente com o fármaco prescrito, ou seja, a dispensação faz parte do processo de promoção da saúde (CONSELHO FEDERAL DE FARMÁCIA, 2016).

O acompanhamento farmacoterapêutico é uma atividade realizada a fim de identificar e resolver os problemas relacionados a farmacoterapia usual, tendo em vista alcançar resultados terapêuticos positivos e consequente qualidade de vida do usuário de medicamentos. Este serviço contribui não só para a redução de problemas acarretados a saúde em virtude do mau uso do medicamento bem como para obter o sucesso do tratamento (SOUZA; BERTONCIN, 2008).

É a partir dos relatos das condições de saúde do paciente é que o profissional farmacêutico irá identificar a necessidade de revisar e corrigir problemas causados pela medicação e então poderá tomar medidas que visem sanar estes problemas, bem como, identificar a falha do próprio paciente com relação a administração do medicamento, podendo assim instruir o mesmo, tendo em vista o sucesso terapêutico (CORRER; OTUKI, 2011).

Já a prescrição farmacêutica é uma prática em que o profissional poderá não só prescrever medicamentos como também recomendar tratamentos alternativos assim como encaminhar pacientes a outros profissionais, tendo como propósito a recuperação da saúde (CFF, 2013a). 
Questão específica 1: A questão específica sobre a RDC 585/2013 teve como objetivo identificar a alternativa incorreta com relação as atribuições do farmacêutico. De um total de dezessete farmacêuticos, quinze $(88,24 \%)$ acertaram a questão, evidenciando o conhecimento por parte dos farmacêuticos com relação as suas atribuições, sendo que dois $(11,76 \%)$ profissionais erraram.

Questão incorreta: "Realizar a prescrição de Medicamentos Isentos de Prescrição (MIPs) e de medicamentos que exijam prescrição". Justificativa: O farmacêutico poderá exercer a prescrição de medicamentos cuja dispensação exija retenção de receita, somente de acordo com prescritores ou instituições de saúde (CFF, 2013b). Porém, dois profissionais responderam como incorretas as duas questões abaixo:

- "Realizar a análise da prescrição medicamentosa quanto a sua adequação aos aspectos legais". Justificativa: É responsabilidade do farmacêutico inspecionar a receita no ato da dispensação do medicamento, verificando se estão descritas todas as informações pertinentes e se esta não se encontra rasurada tendo em vista a proteção à saúde do usuário de medicamentos (CFF, 2013c);

- "Realizar a consulta farmacêutica em local apropriado que proteja as informações do paciente". Justificativa: Segundo a RDC no 585/2013 é de responsabilidade do farmacêutico proceder a consulta em um ambiente que confirme proteção das informações particulares do paciente (CFF, 2013d).

Questão específica 2: A questão específica sobre a RDC 586/2013 teve como objetivo o farmacêutico identificar a questão incorreta. De um total de dezessete farmacêuticos somente quatro $(23,53 \%)$ acerta-

Estação Científica (UNIFAP)

ISSN 2179-1902 ram a questão, evidenciando o déficit por parte dos farmacêuticos com relação ao conhecimento da RDC que regulamenta a prescrição farmacêutica, já que treze $(76,47 \%)$ profissionais erraram.

Questão incorreta: "A prescrição farmacêutica deverá ser realizada unicamente em consultório farmacêutico de modo a garantir a privacidade do paciente no atendimento". Justificativa: De acordo com a RDC 586/2013 a prescrição pode ser exercida em qualquer ambiente, desde que não ocorra a exposição de informações confidenciais do paciente (CFF, 2013b), ou seja, não é necessário um local especial para a realização da prescrição, porém, é imprescindível que o mesmo não permita a livre circulação de pessoas e que as informações repassadas pelo paciente tenham acesso a outrem (CFF, 2013a). Todavia, notou-se que inúmeros profissionais responderam como incorretas as seguintes questões:

- "Os medicamentos dispensados que requerem prescrição médica poderão ser prescritos por farmacêuticos, se preceder de diagnóstico e prenunciado em protocolos e normas técnicas, ou ainda, quando acordado com outros profissionais prescritores ou instituições de saúde". Justificativa: Segundo a RDC 586/2013 o farmacêutico poderá realizar a prescrição de medicamentos que exijam retenção de receita desde que atenda aos requisitos como diagnóstico prévio e que, apresente acordo legal com outros prescritores. Ainda assim, será exigido que o profissional esteja legalizado no Conselho de Farmácia que compete ao estado em que este profissional resida, garantindo ainda que tenha os conhecimentos básicos exigidos para uma prescrição segura e eficiente (CFF, 2013b).

\section{- Para a prescrição farmacêutica de}


medicamentos dinamizados será determinado, que o farmacêutico tenha especialização em Homeopatia. Justificativa: De acordo com a RDC 586/2013 a especialização em homeopatia é um requisito para que se possa exercer a prescrição de forma segura desta classe de medicamentos (CFF, 2016).

Questão específica 3: A questão específica sobre a RDC 586/2013 teve como objetivo identificar a questão correta com relação a prescrição farmacêutica. De um total de dezessete farmacêuticos, doze (70,59\%) acertaram, evidenciando o conhecimento por parte dos farmacêuticos com relação a prescrição farmacêutica, sendo que cinco $(29,41 \%)$ erraram.

Questão correta: "Para desempenhar a função de prescritor o farmacêutico deverá ter domínio e capacitação clínica que incluem boas práticas de prescrição, fisiopatologia, semiologia, comunicação interpessoal, farmacologia clínica e terapêutica". Justificativa: É imprescindível que o profissional tenha amplo conhecimento em determinados temas, visando associar a sintomatologia de determinada doença para então eleger a farmacoterapia pertinente (CRFSP, 2012). Porém, notou-se que inúmeros profissionais responderam como incorretas as questões:

- $\quad$ "Só poderão prescrever farmacêuticos atuantes em farmácias e drogarias". Justificativa: Poderão prescrever os farmacêuticos que se encontram devidamente registrados no Conselho Regional de Farmácia e que apresentem os requisitos mínimos exigidos por este, independente da instituição de saúde que este profissional esteja atuando (CFF, 2016).

- "Poderão ser prescritos todos os medicamentos exceto os homeopáticos".
Justificativa: Poderão ser prescritos todos os MIP's, mesmo os homeopáticos desde que o farmacêutico esteja embasado em estudos referentes a esta classe terapêutica (CFF, 2016).

\section{CONCLUSÃO}

A especialização em farmácia clínica e/ou prescrição farmacêutica é extremamente importante em especial aos profissionais atuantes em farmácias, visto que, é um campo essencial uma vez que, contribui para a promoção da saúde do paciente por meio da prestação dos serviços farmacêuticos, baseada na RDC no 586/2013 do CFF, a qual regulamenta a prescrição farmacêutica.

Mediante a pesquisa é notável que os entrevistados detêm conhecimentos com relação a regulamentação da prescrição farmacêutica, porém, este não se mostrou sólido quando relacionado às questões específicas, principalmente no que diz respeito a conhecer o ambiente em que se deve exercer a prescrição visando os aspectos éticos para com o usuário dos serviços farmacêuticos.

É importante ressaltar que é notável a ausência dos profissionais nos estabelecimentos farmacêuticos e isto, pode implicar em uma má qualidade dos serviços prestados à população Mineirense, fato este que, poderá ser solucionado com uma fiscalização ainda mais efetiva por parte do ConseIho Regional de Farmácia sobre estes profissionais, com a resolução deste problema o farmacêutico poderá ter um reconhecimento notável como profissional da saúde. A ausência destes profissionais pode ser explicada em virtude da não valorização destes profissionais por meio dos baixos salá- 
Conhecimento dos farmacêuticos atuantes em drogarias e farmácias sobre a prescrição farmacêutica na cidade de Mineiros 67

rios pagos a classe, o que desmotiva estes profissionais a permanecerem nos estabelecimentos.

\section{REFERÊNCIAS}

AQUINO, D.S. Por que o uso racional de medicamentos deve ser uma prioridade? Ciência \& Saúde Coletiva, v. 13, p. 733-736, 2008. https://doi.org/10.1590/S1413-81232 $\underline{008000700023}$

BERNARDI, E. A. T. et al. Implantação da avaliação farmacêutica da prescrição médica e as ações de farmácia clínica em um hospital oncológico do sul do Brasil. Espaço para a Saúde-Revista de Saúde Pública do Paraná, Londrina, v. 15, n. 2, p. 29-36, jun. 2014.

BISSON, M.P. Introdução. In: Farmácia Clínica \& Atenção Farmacêutica. 2. ed. Barueri, SP: Manole, 2007. BORTOLON, P. C.; KARNIKOWSKI, M. G.O.; ASSIS, M. Automedicação versus indicação farmacêutica: o profissional de farmácia na atenção primária à saúde do idoso. Revista Atenção Primária à Saúde, v. 10, n. 2, p. 200-209, jul./dez. 2007.

BRASIL. Resolução da Diretoria Colegiada no 44, de 17 de agosto de 2009. Diário Oficial da União. Brasília, n. 134, p. 86, 17 ago. 2009. Seção 1.

BRASILIA. Lei no 13.021, de 8 de agosto de 2014. Diário Oficial da União República Federativa do Brasil, Brasília, 11 ago. 2014. Disponível em: <http://www.planalto.gov. br/ccivil 03/ ato2011-2014/2014/lei/ |13021.htm>. Acesso em: 11 set. 2017. CFF. Conselho Federal de Farmácia. Nota Técnica: Perguntas e respostas referentes ás Resoluções do CFF no 585 e no 586, de 29 de agosto de 2013. Brasília, 2013a. Disponível em: <http://www.cff.org.br/userfiles/fi
le/pdf/Nota\%20T\%C3\%A9cnica\%20585\%20 586.pdf >. Acesso em: 20 fev. 2017.

CFF. Conselho Federal de Farmácia. Regula a prescrição farmacêutica e dá outras providências. Resolução $\mathrm{n}$-586, de 29 de agosto de 2013. Diário Oficial da União, Brasília, n. 187, p. 136, 26 set. 2013b. Seção 1.

CFF. Conselho Federal de Farmácia. Prescrição Farmacêutica e Atribuições Clinicas do Farmacêutico. Brasília, p. 1-26, 2013c. Disponível em: <http://www.cff.org.br/userfil es/prescri\%C3\%A7\%C3\%A30\%20farmaceuti ca\%202015(1).pdf>. Acesso em: 20 fev. 2017.

CFF. Conselho Federal de Farmácia. Regulamenta as atribuições clínicas do farmacêutico e dá outras providências. Resolução no 585, de 29 de agosto de 2013. Diário Oficial da União, Brasília, n. 186, p. 186, 25 set. 2013d. Seção 1.

CFF. Conselho Federal de Farmácia. Programa de suporte ao cuidado farmacêutico na atenção à saúde. Brasília: CFF, 2016.

CORRER, C. J.; OTUKI, M. F. Método clínico de atenção farmacêutica, 2011. Disponível em: <http://www.saude.sp.gov.br/resource s/ipgg/assistencia-farmaceutica/otuki-meto doclinicoparaatencaofarmaceutica.pdf $>$.

Acesso em: 10 set. 2017.

CRF-SP. Conselho Federal de Farmácia do Estado de São Paulo. Área profissional em alta. Revista do Farmacêutico. São Paulo, n. 106, p. 01-60, fev./abr. 2012.

GALATO, D.; ALANO, G.M.; TRAUTHMAN, S.C.; VIEIRA, A.C. A dispensação de medicamentos: uma reflexão sobre o processo para prevenção, identificação e resolução de problemas relacionados à farmacoterapia. Revista Brasileira de Ciências Farmacêuticas, v. 44, n. 3, p. 465-475, jul./set. 2008. https://doi.org/10.1590/S1516-93322 $\underline{008000300017}$ 
FERNANDES, W. S.; CEMBRANELLI, J. C. Automedicação e o uso irracional de medicamentos: o papel do profissional farmacêutico no combate a essas práticas. Revista Univap, São José dos Campos, v. 21, n. 37, p. 05-12, jul. 2015

JOÃO, W.S.J. Serviços farmacêuticos e sociedade: uma mudança de paradigmas. Pharmacia Brasileira, n. 74, p. 44-45, jan./fev. 2010.

JOTZ, G. P. et al. A importância da presença do farmacêutico na farmácia para resolução de problemas básicos de saúde. Revista Associação Médica do Rio Grande do Sul. Porto Alegre, v. 46, n. 3-4, p. 135-138, jul./dez. 2002.

OLIVEIRA, A. B. et al. Obstáculos da atenção farmacêutica no Brasil. Revista Brasileira de Ciências Farmacêuticas, São Paulo, v. 41, n. 4, p. 409-413, out./dez. 2005.

PADUAN, F.; MELLO, J. C. P.; DOBLISNSKI, P. M. F.; DELAPORTE, R. H. Perfil dos profissionais farmacêuticos na cidade de Umuarama - PR. Arquivos de Ciências da Saúde da Unipar, Umuarama, v. 9, n. 1, p. 11-16, jan./abr., 2005.

PEREIRA, L. R. L.; FREITAS, O. A evolução da Atenção Farmacêutica e a perspectiva para - Brasil. Revista Brasileira de Ciências Farmacêuticas, v. 44, n. 4, p. 601-612, out./dez. 2008. https://doi.org/10.1590/ 20S1516-93322008000400006

SANTOS, J.S. O papel social do farmacêutiCo, CFF, Brasília, 2009. Disponível em:<http: //www.cff.org.br/noticia.php?id=182>. Ace sso em 11 set. 2017.

SILVA, S.A. LUCCA, P.S.R. A prescrição farmacêutica: uma análise da percepção dos profissionais farmacêuticos da cidade Cascavel-PR. Revista Brasileira de Ciências Farmacêuticas. Cascavel, 2015. Disponível em: $<$ https://www.fag.edu.br/upload/gradu acao/tcc/55cb87bb166ea.pdf>. Acesso em: 10 set. 2017.

SOUZA, V. V.; BERTONCIN, A. L. F. Atenção farmacêutica para pacientes hipertensosnova metodologia e a importância dessa prática no acompanhamento domiciliar. Revista Brasileira em Promoção da Saúde, Pouso Alegre, v. 21, n. 3, p. 224-230, 2008.

VIEIRA, F.S. Possibilidades de contribuição do farmacêutico para a promoção da saúde. Ciência \& Saúde Coletiva, v. 12, n. 1, p. 213220, 2007. https://doi.org/10.1590/S141381232007000100024

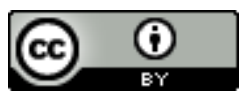

License information: This is an openaccess article distributed under the terms of the Creative Commons Attribution License, which permits unrestricted use, distribution, and reproduction in any medium, provided the original work is properly cited.

Artigo recebido em 11 de outubro de 2017.

Avaliado em 01 de agosto de 2018.

Aceito em 01 de agosto de 2018.

Publicado em 28 de fevereiro de 2019.

\section{Como citar este artigo (ABNT):}

COELHO, Rafaella Ferreira; MACHADO, Fabio Bahls. Conhecimento dos farmacêuticos atuantes em drogarias e farmácias sobre a prescrição farmacêutica na cidade de Mineiros-Goiás. Estação Científica (UNIFAP), Macapá, v. 8, n. 2, p. 57-68, maio/ago. 2018. 